\section{Management of patients on warfarin by general dental practitioners in South West Wales: continuing the audit cycle}

\author{
K. Dewan, ${ }^{1}$ K. Bishop ${ }^{2}$ and A. Muthukrishnan ${ }^{3}$
}

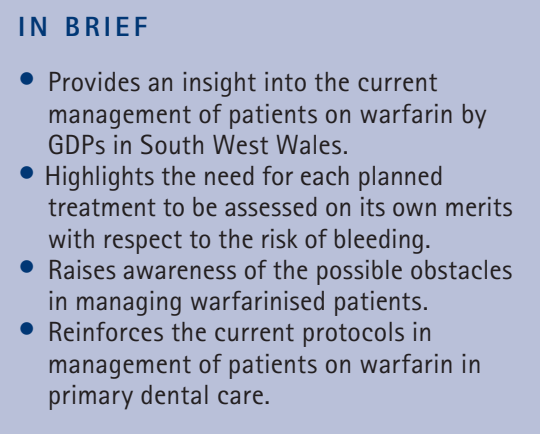
management of patients on warfarin in primary dental care.

Provides an insight into the current management of patients on warfarin by with respect to the risk of bleeding. Raises awareness of the possible obstacles in managing warfarinised patients.

\begin{abstract}
Aims To ascertain the current management protocols of patients on warfarin by general dental practitioners (GDPs) in South West Wales and to compare these findings with current guidelines and the results from a previous audit published in 2003. Materials and methods A questionnaire similar to that used in the first audit was sent to 447 GDPs in South West Wales. In addition, questions were included on factors which might affect international normalised ratio (INR), the timing of pre-operative INR assessment and the risk of bleeding associated with implant surgery. GDPs' details were derived from the online GDC database of registered dental practitioners. Registered specialists and GDPs who practised only orthodontics were excluded. Results Of the 447 questionnaires distributed, $332(74 \%)$ were returned. Eight percent $(n=26)$ of the respondents did not treat patients on warfarin. Two hundred and forty-seven respondents (74\%) considered implant placement as a procedure with high risk of bleeding, with inferior dental block, sub-gingival restorations and sub-gingival debridement receiving a lower response (45\%,28\% and 12\%, respectively). When planning a high risk procedure, 206 respondents (63\%) indicated they would seek advice from a cardiologist or general medical practitioner; none of the respondents would advise the patient to reduce their warfarin dose, while $1 \%$ indicated they would ask the patient to stop taking warfarin without seeking any medical opinion. A total of 278 respondents (84\%) stated they would check the INR before treatment and of these, $214(65 \%)$ indicated they would do so within 24 hours of treatment and $60(18 \%)$ within 48 hours. Ten respondents said they would not normally check INR. One hundred and twelve respondents (34\%) considered 2.5 as the safe upper INR limit for performing high risk procedures, 21 (6\%) considered an INR of between 1 and 2 as the safe limit, 99 (30\%) considered and INR of 3 as safe, 36 (10\%) considered 3.5 as safe and 36 (10\%) considered an INR of 4 as safe. Finally, 286 respondents (86\%) considered drug interactions and 236 (71\%) considered alcohol as significant influencing factors on INR. Conclusions The findings demonstrate a broad change in practice towards the new recommendations produced in 2001 but also highlight that further education and support may be necessary, as well as greater consistency in published guidelines.
\end{abstract}

\section{INTRODUCTION}

Anticoagulation therapy is used to treat patients with a variety of haemostatic disorders in an attempt to prevent thrombus formation. In particular, oral anticoagulants are prescribed for the prophylaxis of thromboembolic disorders, with warfarin the one most widely used in the UK. ${ }^{1,2}$

"Specialist Registrar in Restorative Dentistry, The School of Dentistry, University of Birmingham, St Chad's Queensway, Birmingham, B4 6NN; ${ }^{2}$ Consultant in Restorative Dentistry and Implantology, ${ }^{3}$ Associate Specialist in Restorative Dentistry, Department of Restorative Dentistry, Morriston Hospital, Swansea, SA6 6NL

*Correspondence to: Mr Karun Dewan

Email: karunsinghdewan@ hotmail.com

Tel: +44 (0)121 237 2761; Fax: +44 (0)121 6258815

\section{Online article number E8}

Refereed Paper - accepted 16 October 2008

DOI: $10.1038 /$ sj.bdj.2009.112

${ }^{\oplus}$ British Dental Journal 2009; 206: E8
Warfarin has significant side-effects as a potent vitamin $\mathrm{K}$ antagonist. Warfarin interferes with $\gamma$-carboxylation of factors II, VII, IX and $\mathrm{X}$ as well as the coagulation inhibitor proteins $C$ and S. ${ }^{3}$ The effect of warfarin is monitored using the international normalised ratio (INR), which compares the patient's prothrombin time with a normal control. The INR is only valid for patients on well controlled anticoagulant therapy where the level of anticoagulation is reasonably stable over a period of time. The normal accepted therapeutic range is $2-4 .{ }^{4}$ For each single figure increase in the INR the risk of bleeding is doubled, hence the need to monitor its value.

Since its introduction there has been a debate about the risk of haemorrhage in warfarinised patients requiring dental treatment..$^{5-9}$ As such, a number of protocols have been advocated to reduce the risk by modifying the warfarin regime. These have included substitution, ${ }^{5,6}$ decreasing the levels of anticoagulation, ${ }^{10}$ or even stopping the patient's warfarin for two days pre-operatively. ${ }^{9,11}$ Conversely, concern has been expressed about the increased risk of thromboembolic episodes in patients whose warfarin has been modified to allow dental treatment to occur. ${ }^{8,12-19}$

The wide use of warfarin means that general dental practitioners (GDPs) are exposed to an increasing number of patients on anti-coagulant therapy. Many of these patients require procedures which could be complicated by prolonged coagulation times. A previous survey to assess the management of patients on 
warfarin by GDPs in South West Wales highlighted possible misunderstandings in the appropriate management of such patients in light of recommendations published annually since 2001.,20 This second cycle of the audit aims to re-evaluate the position in a larger number of clinicians and from a wider geographical area.

\section{AIMS}

1. To ascertain the current

management protocols of

patients on warfarin by GDPs

in South West Wales

2. To compare these findings with current guidelines and the results from the previous audit.,21

\section{MATERIALS AND METHODS}

A questionnaire (Appendix 1) was sent to 447 GDPs in South West Wales. This document was similar to that used in the previous audit ${ }^{21}$ other than the question on whether the clinician had any previous hospital experience. This was due to the finding in the original study that these had no effect on decision making.

Questions on factors which might affect the INR, the timing of any preoperative INR assessment and the risk of bleeding associated with implant surgery were included in this audit but not in the original one.

The details of the GDPs were derived from the GDC online database of registered general practitioners using regional postcodes as the search criteria. GDPs or their practices were then contacted to confirm the details. Although this study included practitioners from a larger geographical area than the first audit, the region in the original study was also included in this audit. Clinicians whose practice was limited to orthodontics were not included in the study, nor were any registered specialists. These groups were also omitted from the results of the previous audit. Practitioners were requested to complete the questionnaire anonymously and to return it in a stamped addressed envelope. After four weeks a reminder questionnaire was sent to all the practitioners. Data was then collated and analysed using descriptive methods.

Table 1 GDPs' responses to 'risk of bleeding' assessment for clinical procedures

\begin{tabular}{|l|l|l|l|}
\hline Procedure & $\begin{array}{l}\text { High risk } \\
\text { No. of GDPs (\%) }\end{array}$ & $\begin{array}{l}\text { Low risk } \\
\text { No. of GDPs (\%) }\end{array}$ & $\begin{array}{l}\text { Not noted } \\
\text { No. of GDPs (\%) }\end{array}$ \\
\hline Sub-gingival debridement & $197(59 \%)$ & $116(35 \%)$ & $19(5.7 \%)$ \\
\hline Supra-gingival scaling & $8(2 \%)$ & $304(91 \%)$ & $20(6 \%)$ \\
\hline Sub-gingival restorations & $92(28 \%)$ & $217(65 \%)$ & $23(6.9 \%)$ \\
\hline Periodontal examination & $37(11 \%)$ & $276(83 \%)$ & $19(5.7 \%)$ \\
\hline Inferior dental block (local anaesthetic) & $148(45 \%)$ & $162(49 \%)$ & $22(6.6 \%)$ \\
\hline Infiltration (local anaesthetic) & $11(3 \%)$ & $302(91 \%)$ & $19(5.7 \%)$ \\
\hline Pulp extripation and RCT preparations & $35(11 \%)$ & $277(83 \%)$ & $20(6 \%)$ \\
\hline RCT obturation & $4(1 \%)$ & $307(92 \%)$ & $21(6.3 \%)$ \\
\hline Implant 1st stage procedure & $247(74 \%)$ & $11(3 \%)$ & $74(22 \%)$ \\
\hline Implant abutment connection & $41(12 \%)$ & $215(65 \%)$ & $76(22.8 \%)$
\end{tabular}

\section{RESULTS}

\section{Data collected}

A total of 447 questionnaires were distributed, of which 332 (74\%) were returned. In the previous audit a total of 250 questionnaires were issued with 160 (64\%) returned, of which 155 (62\%) were utilised.

\section{Replies to questions}

\section{GDP's position in practice}

One hundred and ninety-four (58\%) of the respondents were principals, 124 (37\%) were associates and only four (1\%) were vocational dental practitioners (VDPs). Compared with the previous study there was an $11 \%$ reduction in responses from principals and a 3\% reduction in responses from VDPs but a 19\% increase in responses from associates. Of the remaining six respondents, three $(0.9 \%)$ were either locum dentists or on temporary post and the remaining three $(0.9 \%)$ were not known.

\section{Do you treat patients on warfarin?}

In this study a total of 26 GDPs (8\%) did not treat patients on warfarin, with the reasons provided including: 'difficulty in coordinating INR prior to treatment' (2.4\%), 'no support from GP/cardiologist' (1\%), 'potential medico-legal problems' (2.3\%) and 'lack of experience' (2.1\%). This was a similar percentage to the previous study (9\%) and with similar explanations.
Opinion of procedure related to risk of bleeding (Table 1)

GDPs were asked for their opinion regarding the potential risk of bleeding in relation to various common dental procedures. Two hundred and forty-seven respondents (74\%) considered implant placement as a procedure with a high risk of bleeding. The response in relation to inferior dental block and sub-gingival restorations remained almost identical to the original survey (45\% and 28\% respectively). However a marked reduction in the number of practitioners who considered sub-gingival debridement as a high-risk procedure (12\%) was recorded.

\section{Management of anticoagulation}

1. Advice to patients when carrying out a 'high risk of bleeding' procedure. When planning a 'high risk of bleeding' procedure on a patient taking warfarin, a total of 206 of respondents (62\%) indicated that they would seek advice from a general medical practitioner (GMP) or cardiologist. This is comparable to the first study where $67 \%$ of respondents indicated that they would act in a similar fashion. In this study none of the respondents indicated that they would advise the patient to reduce the warfarin dose, while three (1\%) would ask the patient to stop the warfarin without seeking any medical opinion. In the first study 22 (15\%) clinicians advised patients to either stop or reduce their dose of warfarin for one to three days prior to dental treatment without seeking a medical opinion. 
2. INR assessment prior to treatment. A total of $278(84 \%)$ respondents indicated that they would check the INR prior to treatment. This is in comparison to 55\% of GDPs who checked INR prior to treatment in the previous study. Of the total 278 respondents who indicated that they would evaluate the INR prior to any treatment, 218 (65\%) indicated that they would do so within 24 hours of treatment and $60(18 \%)$ within 48 hours. Ten (3\%) said that they would not normally check the INR and 44 (13\%) respondents did not answer this question. The question on the timing of any INR assessment was not included in the first audit since the advice was not published in the Dental practitioners' formulary at the time. ${ }^{22}$

3. Safe upper limit of INR to carry out 'high risk of bleeding' procedures (Fig. 1). One hundred and twelve (34\%) respondents in this study considered 2.5 as the safe upper INR limit for carrying out a procedure with high risk of bleeding, while the equivalent figure from the original study was 51 (36\%). Twentyone $(6 \%)$ of respondents in this study considered that an INR between 1 and 2 was the safe limit compared to 40 (26\%) in the original audit. With respect to an INR of 3 or 3.5, $99(30 \%)$ and $36(10 \%)$ respondents, respectively, considered these levels as safe in this study. In the original study the comparable figures were $11(7 \%)$ and 1 (1\%). In the current study 36 respondents (10\%) considered an INR of 4 as a safe upper limit compared to none in the original survey.

4. Factors influencing patient INR (Fig. 2). A total of 286 respondents (86\%) considered drug interactions and 236 (71\%) considered alcohol as significant influencing factors on the INR. This question was not asked in the previous study.

\section{DISCUSSION}

When anticoagulation therapy was first introduced, the target therapeutic range was a prothrombin ratio (PT/control) of 2.0 to $2.5 .^{13}$ However, due to a lack of standardisation of the estimation of prothrombin time, the degree of anticoagulation used by physicians became higher than the range originally recommended. This obviously increased the risk of adverse bleeding. ${ }^{23,24}$ As such the World Health Organization subsequently

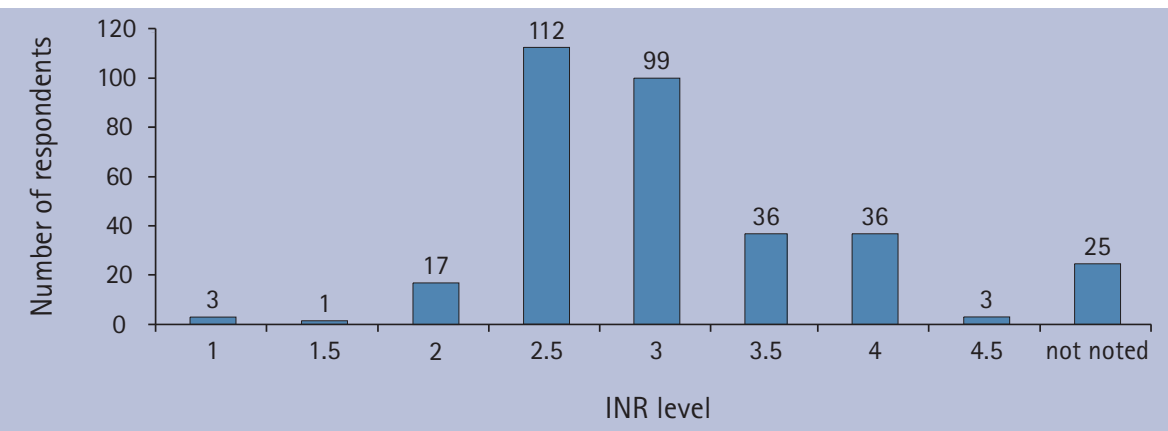

Fig. 1 GDPs' responses to the question on safe INR upper limit

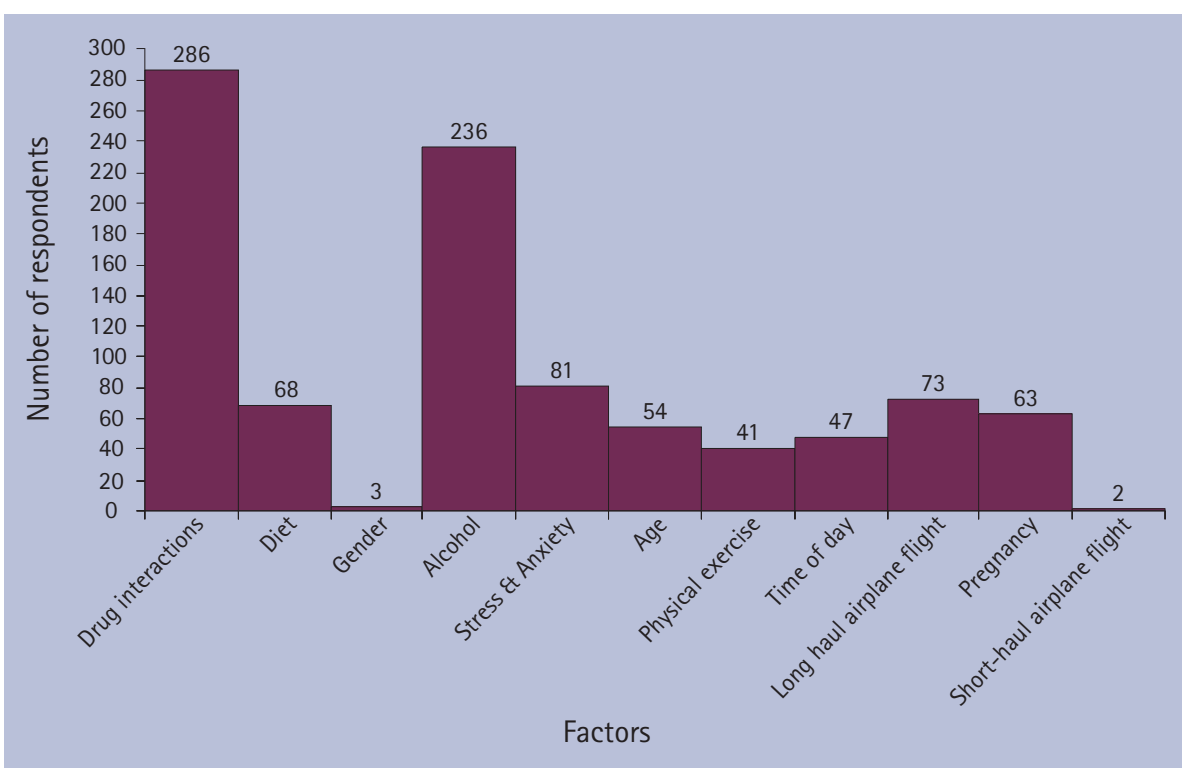

Fig. 2 GDPs' responses to the question on factors that could influence INR

introduced the international normalised ratio (INR) to improve the safety and effectiveness of oral anticoagulation therapy. ${ }^{25}$ The INR adjusts for the varying sensitivities of the thromboplastins and makes it possible to target the same therapeutic ranges while using different laboratory reagents. ${ }^{26}$ In the UK the normal therapeutic range is between 2 and 4 with the exact level dependant on the individual clinician, patient and the condition for which the anticoagulant is prescribed. ${ }^{4}$ With this range and all things being equal, a patient with this degree of anticoagulation would be expected to bleed for two to four times longer than a non-anticoagulated individual. Because of this risk of prolonged bleeding it has been common to discontinue or modify the warfarin treatment prior to dental surgery. The aim of this change in the warfarin regime is to limit post-treatment bleeding problems for procedures associated with a risk of haemorrhage. ${ }^{26,27}$

In 2001 guidelines were produced by the North West Medicines Information
Centre (NWMIC) ${ }^{20}$ on the surgical management of dental patients on warfarin in primary care. NWMIC is a regional unit of the NHS Medicines Information Service which supports the safe, effective and efficient use of medicines by providing up-to-date information and advice. NWMIC specialises in the "surgical management of the primary care dental patient on warfarin'. The service operates to defined national standards and is co-ordinated nationally by the UK Medicines Information Pharmacists' Group. This is an independent and separate body from the British national formulary (BNF) but often uses the BNF as one of its sources of information.

The NWMIC guidelines indicate that the warfarin regime does not need to be routinely changed for those treatments expected to be carried out in general dental practice, providing the INR is stable and not greater than 4 . The guidance therefore assumes that procedures commonly carried out in general dental practice pose a low risk of bleeding. This 
includes minor surgery such as simple extractions of up to three teeth, gingival surgery, crown and bridge procedures, supra-gingival scaling and the surgical removal of a limited number of teeth. ${ }^{1,2,22,28,29}$ However NWMIC suggest that sub-gingival debridement may cause significant bleeding, especially if the tissues are inflamed.

The NWMIC guidelines are based on the following evidence:

- The consensus from reviews on the management of dental patients taking warfarin

- Continuing warfarin during dental surgical procedures may increase the risk of postoperative bleeding requiring intervention. However most cases of postoperative bleeding are easily treated with local measures such as packing with a haemostatic dressing, suturing and pressure

- Stopping warfarin increases the risk of thromboembolic events; the risk of thromboembolism after withdrawal of warfarin therapy outweighs the risk of oral bleeding, as bleeding complications, while inconvenient, do not carry the same risks as thromboembolic complications

- Stopping warfarin is no guarantee that the risk of postoperative bleeding requiring intervention will be eliminated, as serious bleeding can occur in non-anticoagulated patients.

Further guidelines regarding the management of patients on oral anticoagulants requiring dental surgery have been produced by a committee consisting of the British Society for Haematology (BSH), the British Dental Association (BDA) and the National Patient Safety Agency (NPSA). ${ }^{30}$ This group also concluded that the risk of significant bleeding in patients on oral anticoagulants with a stable INR of between 2-4 is small. As such they recommended that oral anticoagulants should not be discontinued in the majority of patients requiring out-patient dental surgery, including extractions. In addition, they also recommended the use of local measures such as oxidised cellulose, sutures, sponges and 5\% tranexamic acid mouthwash to minimise the risk of bleeding in patients undergoing dental surgery.
The BNF, which is a joint publication of the British Medical Association and the Royal Pharmaceutical Society of Great Britain, has also produced guidelines on managing warfarin in dental practice. ${ }^{31}$ Although these recommendations are broadly similar to those produced by NPSA/BDA/BSH and NWMIC, they differ in two important areas. The BNF suggests that if an extraction is required then the acceptable upper level for the INR is 3 , while 4 is appropriate for all other minor dental procedures.

However, an INR upper limit of 4 appears to be supported by studies which have examined the implications of accepting this level when carrying out minor surgical procedures..$^{1,2,22,32,33}$ For example a randomised controlled trial of 57 anticoagulated patients found no increase in clinically important bleeding, with only one patient requiring admission to hospital for observation after closure of an oral-antral communication. Nine patients who had delayed bleeds were treated at home following the provision of simple advice over the telephone. ${ }^{32}$ Conclusions from a recently published case series study $^{33}$ of 150 patients on warfarin also support the view that provided the INR is between 2-4, extractions can be carried out in primary care without the risk of a significant bleed.

The BNF also recommends that the medical practitioner should be consulted for all patients who are on long-term oral anticoagulant therapy, irrespective of the stability of the INR. In contrast, the NWMIC advises that a medical opinion is only necessary when the INR requires modification or is unstable.

The BNF guidelines provide dentists with an authoritative and easily accessible source of information. As such, GDPs are likely to use this publication as their first source of guidance. However, in the case of managing warfarinised patients, it appears that the BNF guidance, although in broad agreement with comparable publications, may be at variance in specific and important areas.

\section{Cohort and response}

The cohort in this study was from a greater geographical area than in the original audit but included the original region. This expansion allowed a larger number of practitioners to be involved while still including those practitioners who may have been included in the original study. No attempt was made to evaluate how many of the practitioners who responded to the initial questionnaire replied to the current one. As such, it is possible that the original study and its findings were based on an atypical group. However, considering the numbers involved it is unlikely that this is the case and as such, a comparison between the two studies can be made. Increasing the numbers in the current audit further reinforces the validity of the findings and conclusions.

The response rate of this audit (74\%) was greater than the original study, which in itself was considered good. This is perhaps an indication of increased awareness and understanding among general dental practitioners in the management of patients on oral anticoagulants and therefore a willingness to contribute to the study. It may also reflect the thoroughness of the database used to identify the practitioners. The response rate also suggests that regional audits may be a valid method of assessing knowledge and awareness of general dental practitioners. ${ }^{34}$

It was not surprising to find that only four VDPs responded to the questionnaire. South West Wales has a programme which involves only 12 VDPs each year. In addition, this low response may be a reflection of this group of practitioners' lack of confidence or experience in managing patients on anticoagulant therapy. If this is the case then this finding may be of benefit to those responsible for planning undergraduate and VDP training.

Another interesting finding was the 11\% decrease in replies from principals and an $18 \%$ increase in response from associates. This could be a reflection of the changing ratio of these groups in general dental practice or an increase in confidence and knowledge of associates in managing patients on anticoagulant therapy. The uncertainty prior to the introduction of the new GDP contract may also have contributed to this result as associates may have preferred to preserve their existing status rather than make the financial commitment associated with becoming a principal. With the introduction of the new contract in 2006, future audits may 
see the reverse occur due to the way units of dental activity are allocated.

\section{Treatment}

The number of GDPs who would not treat patients on warfarin remained low but, disappointingly, largely unchanged between the two audits ( $8 \%$ in 2007 compared to $9 \%$ in 2001). This suggests that there is a core of GDPs who are reluctant to treat this patient group. The reasons provided for not treating are also largely unchanged since the initial audit and appear to be based on a lack of appropriate medical support to manage changes in the warfarin regime. This is disappointing since the current guidelines suggest that a change in the warfarin regime or medical support are not routinely required. As such this finding suggests a lack of understanding or awareness of the new recommendations. An alternative explanation is that despite the guidelines, some practitioners feel unhappy with managing prolonged bleeding even if this is limited and to be expected.

\section{Risk of bleeding}

There is limited detailed documented guidance on procedures which are considered to have either a high or low risk of bleeding. The majority of guidelines and recommendations $s^{4,20,27,32,33}$ have focused on surgical aspects of dentistry. There has only been limited information available on the management of patients requiring restorative treatment. ${ }^{25,26}$

For example, the consensus committee of the BSH, BDA and NPSA ${ }^{30}$ suggested that the following procedures did not require any evaluation of the INR prior to commencing treatment, as the risk of bleeding was low:

- Scaling and polishing in the presence of CPITN score 1

- Conservative procedures such as direct and indirect restorations

- Construction of fixed and removable prostheses.

The most recent NWMIC guidelines ${ }^{4}$ make the broad assumption that the care provided by GDPs in a primary care setting is unlikely to involve treatments with a high risk of haemorrhage. The NWMIC concluded that up to three extractions in the same quadrant would be associated with a low risk of bleeding. They had a similar view on periodontal examinations and supra-gingival scaling but considered sub-gingival debridement as a high risk treatment if the tissues are inflamed.

A notable number of respondents in this audit (41\%) did not consider subgingival debridement a high risk bleeding procedure and this had increased from the previous survey (29\%). Initially this result appears surprising considering the guidance available. However, as highlighted by the NWIMC, the risk of bleeding from sub-gingival debridement is determined by a number of factors, of which the degree of inflammation is obviously an important consideration. In situations where there is minimal inflammation but persistent pocketing in excess of 4-5 $\mathrm{mm}$, as can occur in maintenance periodontal therapy, then the risk of bleeding may not be great.

However, sub-gingival instrumentation has the potential to cause significant bleeding in warfarinised patients if the periodontal tissues are inflamed. As such, in these circumstances it is important to minimise the degree of inflammation prior to commencing the sub-gingival treatment. This may be achieved by encouraging good plaque control, the short-term use of a chlorhexidine mouthrinse and initially carrying out simple supra-gingival scale and polishing. Any subsequent, subgingival scaling and debridement can also be restricted to a limited area, eg one quadrant. The long-term aim in warfarinised patients, in common with other susceptible groups, is to maintain a routine high level of oral hygiene and access to regular screening and support.

It would appear therefore that each case has to be assessed on its own merits regarding the risk of haemorrhage and the need to modify the INR. This is supported by the finding in relation to implant therapy.

The majority of respondents (74\%) considered first stage implant surgery to present a high risk of bleeding. However, a single implant placement may be associated with no greater risk of bleeding than an extraction. This may be especially the case if the fixture is inserted using a flapless technique which decreases the amount of invasion and minimises the risk of haemorrhage. However, these techniques are demanding and require careful pre-operative planning and a good three-dimensional awareness to visualise the underlying tissues. If these are not achieved then the risk of bleeding due to inappropriate site preparation may be high.

Conversely, conventional dental implant placement requires the raising of a mucoperiosteal flap, drilling into and often through the alveolar bone prior to fixture placement. This can pose an increased risk of bleeding for patients on oral anticoagulants, particularly if multiple fixtures are inserted, and as such it can be considered a 'high risk of bleeding' procedure.

\section{Management of anticoagulation}

It was encouraging to note that in the current audit only three (1\%) dentists would stop a patient's medication without seeking medical opinion. This was a notable improvement from the original study where $15 \%$ of the respondents would either stop or change the oral anticoagulant dose. This change would suggest that GDPs are now more aware of the guidelines and the risk of thromboembolic events occurring with either altering or stopping anticoagulant therapy.

This conclusion is also supported by the finding that $84 \%$ of GDPs would evaluate the patient's INR prior to dental treatment compared with 54\% in the previous audit. The majority (66\%) of the respondents within this group would evaluate the INR within 24 hours.

An evaluation within 24 hours of treatment probably provides the most accurate assessment of the INR status. However, if the warfarin dose requires adjustment, an assessment at this time does not allow modification of the regime to take place before the treatment date. In such circumstances, invariably the treatment has to be delayed. For this reason, both the NWMIC and BNF suggest that where the INR is stable (which should be the case if the patient is to be treated in general dental practice) then a check can be carried out up to 72 hours before the planned date of treatment. This will allow any necessary changes in the warfarin dose to be instigated and re-evaluated before the date of treatment. 
Findings from this audit, where 30\% considered 3.0 as a safe upper limit and only $10 \%$ considered this to be 4.0 , suggest that either GDPs are unaware of the NWMIC guidelines or are using the BNF as their only source of information. Even so, 75 (23\%) GDPs still considered an INR greater than 3.0 as unacceptable, which is not in line with any officially published guideline at the time of the survey. Although this is an improvement on the first study, where only $8 \%$ considered an INR $\geq 3.0$ as being the safe upper limit, the results are disappointing considering the new limits have been in existence for at least six years. This finding would suggest that many patients are still having unnecessary adjustments made to their warfarin regime or are being referred to hospitals for their care. The former means that this group has an increased risk of a thromboembolic events occurring, as well as requiring unnecessary haematological investigations. With respect to the unnecessary referrals, this places increased demand on secondary care resources and will invariably mean more inconvenience for patients due to the need to travel to hospitals and being unable receive treatment near their home.

Although this study suggests that more GDPs are managing patients with INR values of 2.5 and 3.0, there appears to be an inherent reluctance to accept INR values greater than 3.0. These levels were those recommended as acceptable upper limits prior to the recent guidelines. This would suggest that some of the respondents are either still unaware of the recent guidelines or are not confident with the recommendations.

\section{Factors affecting the INR}

The objective of this question was to evaluate whether practitioners were broadly aware of factors which could affect the INR.

The majority of respondents considered drug interactions (86\%) and alcohol (71\%) as key factors which could affect the INR. With respect to drugs, no attempt was made to identify which specific drugs they considered since it was felt this would encourage clinicians to seek further information before replying.

Aspirin and the non-steroidal anti- inflammatory drugs (NSAIDs) are perhaps the best known for inhibiting the clotting process and therefore potentially raising the INR of warfarinised patients. Other commonly used antibiotics, such as metronidazole or the macrolides, will greatly increase the effect of warfarin by reducing its metabolism. Other broadspectrum antibiotics can also reduce the amount of the normal bacterial flora in the bowel, which may affect the absorption of vitamin K..$^{35}$ These groups of drugs are probably the ones most likely to be prescribed by GDPs, particularly in acute circumstances. As such it was reassuring to note that the majority of respondents considered drug interactions as a possible factor which could affect the INR.

Interestingly, $71 \%$ of respondents also considered alcohol as a factor which could affect the INR. The interaction between alcohol and warfarin is complex. In general, alcohol has a vasodilatory effect and can increase bleeding. In addition, chronic heavy alcohol intake potentiates liver enzymes which increase the metabolism of warfarin. This leads to lower circulating levels of warfarin. However, chronic liver disease itself can increase bleeding due to its negative effect on vitamin K-dependant clotting factors. ${ }^{36}$ No attempt was made in this audit to assess whether practitioners considered alcohol to have a negative or positive effect on the INR.

It was interesting to note that 24\% and $21 \%$ of respondents considered stress and long-haul aeroplane flights, respectively, as influencing factors on INR. This was a slightly greater number than those who thought diet (20\%) was a factor.

There is no evidence that stress or long-haul flights affect the INR but food that contains large quantities of vitamin $\mathrm{K}$, such as root and leafy green vegetables, will reduce the warfarin effect. Conversely, low levels of vitamin K can lead to reduced prothrombin, which may result in delayed clotting and increase the effect of warfarin.

\section{CONCLUSION}

This audit has provided an insight into the current management of patients on warfarin by general dental practitioners in South West Wales. It has highlighted in general an increasing compliance with guidelines which have been in existence since 2001. However, there appears to be a core of individuals who are either unaware of the changes or are resistant to changing their practice. Conflicting advice in guidelines issued by the BNF and NWMIC may contribute to this outcome. To overcome these inconsistencies, bodies responsible for undergraduate and postgraduate education may need to consider further educational initiatives. In addition, authorities which produce guidelines in this area may need to ensure consistency in the future.

This study also highlights the need for each planned treatment to be assessed on its own merits with respect to the risk of bleeding, with consideration given to the existing published guidelines. In addition, it is important that the clinician has a clear understanding of the tissues involved, the potential affects of any invasive treatment and how optimum haemostasis is achieved.

1. Lockhart P B, Gibson J, Pond S H, Leitch J. Dental management considerations for the patient with an acquired coagulopathy. Part 2: coagulopathies from drugs. Br Dent J 2003; 195: 495-501.

2. Webster K, Wilde J. Management of anticoagulation in patients with prosthetic heart valves undergoing oral and maxillofacial operations. Br J Oral Maxillofac Surg 2000; 38: 124-126.

3. Guidelines on oral anticoagulation: third edition. Br J Haematol 1998; 101: 374-387.

4. North West Medicines Information Centre. Surgical management of the primary care dental patient on warfarin. Liverpool: North West Medicines Information Centre, 2007.

5. Roser S M, Rosenbloom B. Continued anticoagulant in oral surgery procedures. Oral Surg Oral Med Oral Pathol 1975; 40: 448-457.

6. Madura J A, Rookstool M, Wease G. The management of patients on chronic coumadin therapy undergoing subsequent surgical procedures. Am Surg 1994; 60: 542-546.

7. Wahl M J, Howell J. Altering anticoagulation therapy: a survey of physicians. J Am Dent Assoc 1996; 127: 625-638.

8. Devani P, Lavery K M, Howell C J T. Dental extractions in patients on warfarin: is alteration of anticoagulant regime necessary? Br J Oral Maxillofac Surg 1998; 36: 107-111.

9. Mulligan $R$, Weitzel K G. Pretreatment management of the patient receiving anticoagulant drugs. J Am Dent Assoc 1988; 117: 479-483.

10. Johnson W T, Leary J M. Management of dental patients with bleeding disorders: review and update. Oral Surg Oral Med Oral Pathol 1988; 66: 297-303.

11. Donoff R B. Massachussetts general hospital manual of oral and maxillofacial surgery. pp 100 102, 152. St Louis: CV Mosby, 1987.

12. Wahl M J. Dental surgery in anticoagulated patients. Arch Intern Med 1998; 158: 1610-1616.

13. Kovich 0 , Otley C C. Thrombotic complications related to discontinuation of warfarin and aspirin therapy perioperatively for cutaneous operation. J Am Acad Dermatol 2003; 48: 233-237.

14. Caliendo F J, Halpern V J, Marini C P et al. Warfarin anticoagulation in the perioperative period: is it safe? Ann Vasc Surg 1999; 13: 11-16.

15. Blacker D J, Wijdicks E F M, McClelland R L. Stroke risk in anticoagulated patients with atrial 
fibrillation undergoing endoscopy. Neurology 2003; 61: 964-968.

16. Yasaka M, Naritomi H, Minematsu K. Ischemic stroke associated with brief cessation of warfarin. Thromb Res 2006; 118: 290-293.

17. Dunn A S, Turpie A G G. Perioperative management of patients receiving oral anticoagulants. Arch Intern Med 2003; 163: 901-908.

18. Webster K, Wilde J. Management of anticoagulation in patients with prosthetic heart valves undergoing oral and maxillofacial operations. Br J Oral Maxillofac Surg 2000; 38: 124-126.

19. Weibert R T. Oral anticoagulant therapy in patients undergoing dental surgery. Clin Pharm 1992; 11: 857-864.

20. North West Medicines Information Centre. Surgical management of the primary care dental patient on warfarin. Liverpool: North West Medicines Information Centre, 2001

21. Muthukrishnan A, Bishop K. An assessment of the management of patients on warfarin by general dental practitioners in South West Wales. Br Dent $J$ 2003; 195: 567-570

22. Alexander R, Ferretti A C, Sorensen J R. Stop the nonsense not the anticoagulants: a matter of life and death. NYState Dent J 2002; 68: 24-26.
23. Fiore L, Deykin D. Anticoagulant therapy. In Beutler E, Lichtman M A, Coller B S, Kipps T J (eds) Hematology. 5th ed. pp 1562-1584. New York: McGraw-Hill, 1995.

24. Bussey H I, Force R W, Bianco T M, Leonard A D. Reliance on prothrombin time ratios causes significant errors in anticoagulation therapy. Arch Intern Med 1992; 152: 278-281.

25. Herman W W, Konzelman J L, Sutley S H. Current perspectives on dental patients receiving coumarin anticoagulant therapy. J Am Dent Assoc 1997; 128: 327-335.

26. Chugani V. Management of dental patients on warfarin therapy in a primary care setting. Dent Update 2004; 31: 379-384.

27. Randall C. Surgical management of the primary care dental patient on warfarin. Dent Update 2005; 32: 414-426.

28. Scully C, Cawson R A. Medical problems in dentistry. 5th ed. pp 148-151. Edinburgh: Elsevier Churchill Livingstone, 2005.

29. Scully C, Wolff A. Oral surgery in patients on anticoagulant therapy. Oral Surg Oral Med Oral Pathol Oral Radiol Endod 2002; 94: 57-64.

30. Perry D J, Noakes T J C, Helliwell PS. Guidelines for the management of patients on oral anticoagulants requiring dental surgery. Br Dent $J$ 2007; 203: 389-393.

31. British national formulary 52. pp 24-29. London: RPSGB/BMA, 2007.

32. Evans I L, Sayers M S, Gibbons A J, Price G, Snooks $\mathrm{H}$, Sugar A W. Can warfarin be continued during dental extraction? Results of a randomized controlled trial. Br J Oral Maxillofac Surg 2002; 40: 248-252.

33. Salam S, Yusuf $\mathrm{H}$, Milosevic A. Bleeding after denta extractions in patients taking warfarin. Br J Oral Maxillofac Surg 2007; 45: 463-466.

34. Bero L A, Grilli R, Grimshaw J M, Harvey E, Oxman A $D$, Thomson M A. Closing the gap between research and practice: an overview of systematic reviews of interventions to promote the implementation of research findings. The Cochrane Effective Practice and Organization of Care Review Group. BMJ 1998; 317: 465-468

35. Holbrook A M, Pereira J A, Labiris R et al. Systematic overview of warfarin and its drug and food interactions. Arch Intern Med 2005; 165: 1095-1106.

36. Tatsumi A, Kadobayashi M, Iwakawa S. Effect of ethanol on the binding of warfarin enantiomers to human serum albumin. Biol Pharm Bull 2007; 30: 826-829.

\section{Appendix 1 The study questionnaire}

1. Position (check $(\checkmark)$ only one):
$\square$ Principal
$\square$ Assistant
$\square$ Other (specify)
$\square$ Associate
$\square$ Vocational dental practitioner

2. Number of years since qualification? Years

3. Please indicate below if you treat patients on warfarin (check $(\checkmark)$ only one):

$\square$ Yes $\square$ No

4. If 'Yes' please proceed to question 5.

If your answer is ' $\mathrm{No}$ ' then please indicate the reason below and then return the questionnaire using the SAE provided.

$\square$ Time consuming $\quad \square$ No support from GP/cardiologist $\quad \square$ Not sure about the regulations
$\square$ Difficulty in coordinating INR prior to treatment
$\square$ Financial
$\square$ Potential medico-legal problems

Other (specify)

5. In your view in patients on warfarin, what is the risk of bleeding in relation to the procedures listed below (check ( $\checkmark$ ) High or Low for each):

Procedure

Sub-gingival debridement

Supra-gingival scaling

Inferior dental block

Infiltration

Sub-gingival restorations

Periodontal examinations

Pulp extirpation and RCT preparation

RCT obturation

Implant 1st stage procedure

Implant abutment connection

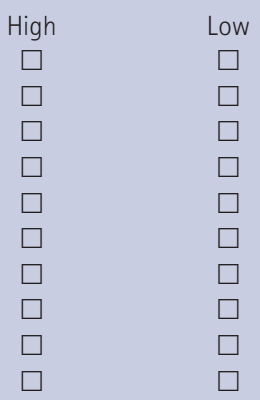

6. When planning a 'high risk of bleeding' procedure on a patient taking warfarin, please indicate your normal advice to the patient

(check $(\checkmark)$ only one):

$\square$ Stop warfarin dose without seeking a medical opinion

$\square$ Other (please provide details)

Low

$\square$

$\square$

7. If you normally check the INR of a patient requiring a 'risk of bleeding' procedure, when would you carry this out? (check ( $\checkmark$ ) only one):

$\square$ Within 24 hours of treatment $\quad \square$ Within 48 hours

$\square$ Would not normally check the INR $\quad \square$ Other (specify)

8. Please indicate what you would consider to be the safe upper limit of INR for carrying out 'high risk of bleeding' procedures.

(Please circle the value below):
1.0
1.5
2.0
2.5
3.0
3.5
4.0
4.5
5.0

9. Which of the following factors do you think could significantly influence the INR? (check $(\checkmark$ ) one or more):
$\square$ Drug interactions
$\square$ Stress \& anxiety
$\square$ Long-haul airplane flight
$\square$ Diet
$\square$ Age
$\square$ Gender
$\square$ Physical exercise
$\square$ Short-haul airplane flight
$\square$ Alcohol
$\square$ Time of day

Thank you for agreeing to participate in this project.

Please return the completed questionnaire in the SAE provided 